\title{
Flexible free-standing SU-8 microfluidic impedance spectroscopy sensor for 3-D molded interconnect devices application
}

\author{
Marc-Peter Schmidt ${ }^{1}$, Aleksandr Oseev ${ }^{1}$, Christian Engel $^{2}$, Andreas Brose ${ }^{1}$, Bertram Schmidt ${ }^{1}$, and \\ Sören Hirsch ${ }^{3}$ \\ ${ }^{1}$ Institute of Micro and Sensor Systems, Otto von Guericke University Magdeburg, \\ Universitätsplatz 2, 39106 Magdeburg, Germany \\ ${ }^{2}$ TEPROSA GmbH, Paul-Ecke-Str. 6, 39114 Magdeburg, Germany \\ ${ }^{3}$ Department of Engineering, University of Applied Sciences Brandenburg, Magdeburger Str. 50, 14770 \\ Brandenburg an der Havel, Germany
}

Correspondence to: M.-P. Schmidt (marc-peter.schmidt@ovgu.de)

Received: 1 July 2015 - Revised: 16 December 2015 - Accepted: 1 February 2016 - Published: 25 February 2016

\begin{abstract}
The current contribution reports about the fabrication technology for the development of novel microfluidic impedance spectroscopy sensors that are directly attachable on 3-D molded interconnect devices (3DMID) that provides an opportunity to create reduced-scale sensor devices for 3-D applications. Advantages of the MID technology in particular for an automotive industry application were recently discussed (Moser and Krause, 2006). An ability to integrate electrical and fluidic parts into the 3D-MID platform brings a sensor device to a new level of the miniaturization. The demonstrated sensor is made of a flexible polymer material featuring a system of electrodes that are structured on and embedded in the SU-8 polymer. The sensor chips can be directly soldered on the MID due to the electroless plated contact pads. A flip chip process based on the opposite electrode design and the implementation of all fluidic and electrical connections at one side of the sensors can be used to assemble the sensor to a three-dimensional substrate. The developed microfluidic sensor demonstrated a predictable impedance spectrum behavior and a sufficient sensitivity to the concentration of ethanol in deionized water. To the best of our knowledge, there is no report regarding such sensor fabrication technology.
\end{abstract}

\section{Introduction}

Impedance spectroscopy is a well-known method for liquid analysis (Barsoukov and Macdonald, 2005). For decades it is applied in microfluidic systems for determination of liquid properties (Gawad et al., 2001; Gómez et al., 2001). Depending on the microchannel material, microfluidic sensors can be fabricated on the wafer level (Schmidt et al., 2014a, b) or they can be completed as a free-standing structure for the attachment on a 3-D substrate. There are a number of advantages when microfluidic sensors are implemented on the wafer level, but at the same time they remain non-applicable for the usage within a 3-D-shaped system.

During the last years, molded interconnection devices (MIDs) have attracted considerable attention. Several advan- tages applicable for the automotive industry such as a high level of integration ability, a compact packaging, etc. were recently highlighted (Moser and Krause, 2006). The possibility of applying 3D-MID-based sensor systems for in-line gasoline analysis can raise considerable interest especially regarding the control of ethanol-containing fuels (Oseev et al., 2013). Structures completed within the MID technology can be applied as a detail of a 3-D system and simultaneously have directly all required integrated electrical and fluidic interconnections. Due to the injection molding and metallization process, a wide range of 3-D-shaped substrates can be fabricated (an example is shown in Fig. 1). In order to be applied in such 3-D systems, the sensor should have a flexible origin with the further possibility of a 3-D direct MID attachment. 


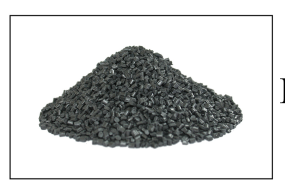

Thermoplastic LDS-pellets

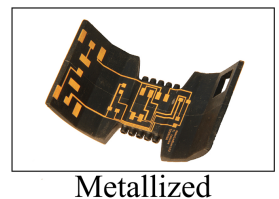

MID-substrate
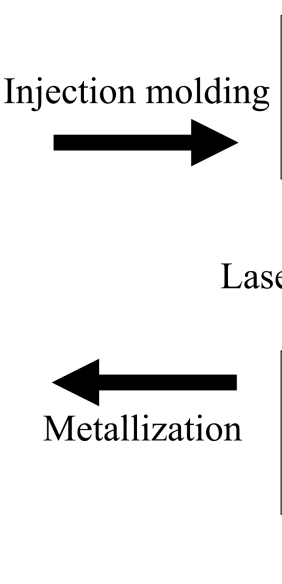

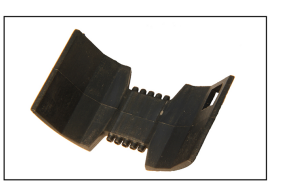

MID-substrate Laser activation

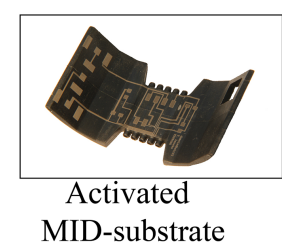

Figure 1. Manufacturing process of a 3D-MID substrate with fluidic channels and conducting paths fabricated by laser direct structuring (LDS) (Schmidt et al., 2012).

The manufacturing of free-standing microfluidic structures can be completed under different approaches (Liu, 2007). Polydimethylsiloxane (PDMS) is well known as a material for the manufacturing of microfluidic structures (Jo et al., 2000). It is a considerably low-cost material, but it cannot be directly structured in a photolithography process. In order to structure PDMS, different techniques such as micromolding need to be applied. These processes can considerably affect the resolution of the final microfluidic structures.

SU-8 is a well-known photoresist that is widely used as a construction material for microfluidic structures, sensors, and a variety of different applications. Based on EPONResin, SU-8 is chemically stable and an optically transparent material with controllable mechanical properties through its processing and possibility of being directly structured with a standard photolithography process (Lorenz et al., 1997; Despont et al., 1997).

A variety of wafer-based SU-8 microfluidic structures have already been published (Lin et al., 2002; Zhang et al., 2001), but completely free-standing SU-8 microfluidic structures are rarely declared due to the complex structuring process of metal layers on SU-8 and the releasing step technology for centimeter-scale structures.

In Vilares et al. (2010), the fabrication of a SU-8-based thermal flow sensor with a manual Kapton film releasing was demonstrated. The releasing process was completed only from the single side of the fluidic channel and finalized with the pure SU-8 encapsulation of microfluidic structures. After the releasing step, the entire microfluidic sensor still remained on a second PMMA handling wafer; therefore, it was not applicable for the 3-D attachment. The same Kapton film releasing steps were used in Ezkerra et al. (2007) where SU8 free-standing cantilever structures were embedded in SU8/Si-based structures and in Agirregabiria et al. (2005) where SU-8 multilayer microstructures were adhesive bonded and released with the help of a Kapton film. In all referred contributions the final microfluidic structures remained on a handling wafer that makes them inapplicable for a threedimensional attachment. Additionally to that, the Kapton foil is unsuitable to be a whole wafer releasing solution because it causes a considerable mechanical stress. In some cases, this mismatch can completely destroy the free-standing SU8 structures.

Recently, the new nanoscale sacrificial releasing layer Omnicoat $^{\mathrm{TM}}$ was introduced and applied for SU-8 structure releasing (Wang et al., 2009). It was initially aimed to be applied for stripping of SU-8 molds in which metal structures are electroplated, so it has become a very convenient and considerably clean method for the wet releasing of SU-8 microfluidic structures (Pesantez et al., 2008). In the current contribution, we demonstrate a SU-8-based technology with an Omnicoat ${ }^{\mathrm{TM}}$ releasing step that allows completely freestanding SU-8-based microfluidic sensors.

In comparison to any previously published results, we demonstrate an impedance spectroscopy sensor with integrated opposite metal electrode structures in SU-8 and released from both sides to achieve a free-standing and flexible SU-8-based sensor. The manufactured free-standing SU8 microfluidic sensor can be used for a subsequent 3-D-MID attachment that provides an opportunity to create reducedscale sensor devices for 3-D applications.

\section{3D-MID interposer}

The microfluidic interposer that has a three-dimensional shape was injection molded in an Arburg 320 S 500. As molding material, the thermoplastic liquid crystal polymer Vectra E840i LDS was used. It also can be directly applied for a laser direct structuring (LDS) (Leneke et al., 2009). The polymer material implies an additive of a metal-organic copper-based complex. This complex can be split into metal atoms (surface activation) which act as a nucleus in a chemical copper plating by a focused IR-laser beam. For this reason, the LPKF MicroLine 3-D 160i system was used for the laser structuring of the MID surface. The Nd: YAG laser with a wavelength of $1064 \mathrm{~nm}$ was utilized. The following laser parameters were applied for the process: power $2.5 \mathrm{~W}$, speed $2000 \mathrm{~mm} \mathrm{~s}^{-1}$, frequency $100 \mathrm{kHz}$, and a spot size of $60 \mu \mathrm{m}$ with a writing overlap of $50 \%$. The complete LDS technological process is shown in Fig. 1.

After the laser structuring of the polymer, a mechanical precleaning step was performed to eliminate residuals from the surface. By electroless plating of copper, nickel and gold on the irradiated structures a solderable surface was achieved. This metallization process contains four main steps. At first, an intensive cleaning step removed any pollution on the surface in an alcohol-based solution with the help of an ultrasonic bath. Further, a first metallization bath of copper(II) sulfate, Cupralux INI, was performed in order 


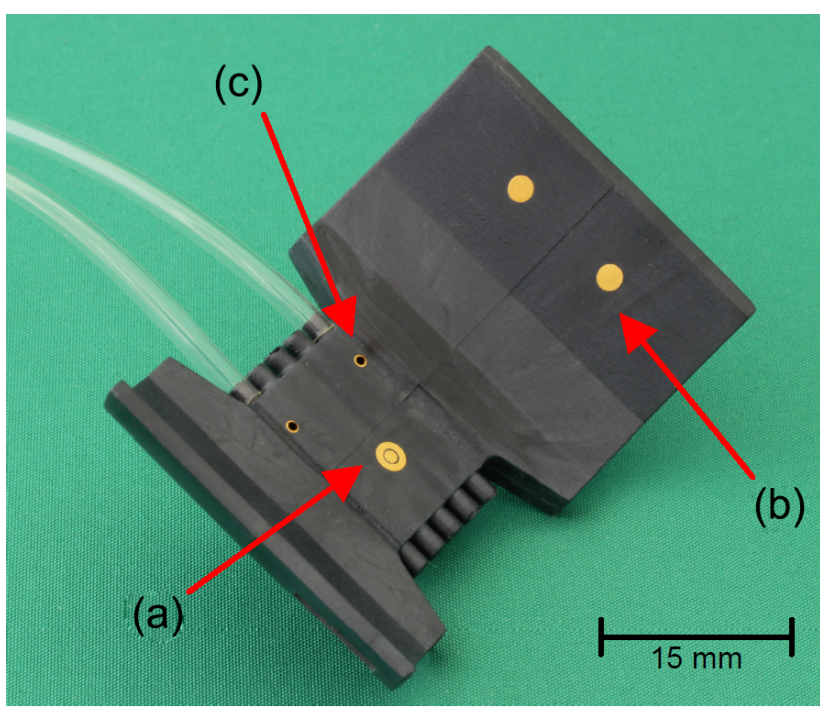

Figure 2. 3D-MID carrier for the flexible impedance spectroscopy microfluidic sensor; central sensor contact (a), mechanical stabilization pads (b), and fluidic contact (c).

to deposit a 5-7 $\mu \mathrm{m}$ thick copper layer on the substrate to ensure a proper conductivity of the metallization. Then, after short treatment in a palladium chloride solution, the surface of the plated copper was catalyzed for a subsequent nickel layer deposition. The MID Nickel HP solution was used for the following nickel plating step. It was heated up to $83^{\circ} \mathrm{C}$, which enabled reaching a nickel layer with a thickness of a minimum of $5 \mu \mathrm{m}$ atop of the previously plated copper within $40 \mathrm{~min}$. The metallization finish was performed by an immersion gold bath of the MID Gold MP to grow a $0.1 \mu \mathrm{m}$ layer atop to prevent any possible oxidation of the free nickel surface. The completed plating process of the MID guaranties the compatibility with standard packaging technologies such as flip chip and lead free soldering.

The three-dimensional injection molded device has fluidic channels with a diameter of $1.5 \mathrm{~mm}$ and a length of $20 \mathrm{~mm}$ for the fluidic supply to provide an easy attachment of the commercial tubes (Fig. 2). To establish a connection between the fluidic channels of the MID platform and the microfluidic test chip two ring-shaped solder connections define a sealed fluidic interconnection with an inner diameter of $500 \mu \mathrm{m}$ on the MID surface. The fluidic ports were laser drilled with the same 160i IR laser system in a spiral form and a rise of $30 \mu$ rotation $^{-1}$ but with different laser parameters. The used laser power was increased up to $4.5 \mathrm{~W}$; the speed and frequency were lowered to $500 \mathrm{~mm} \mathrm{~s}^{-1}$ and $50 \mathrm{kHz}$. Besides the fluidic ports, a circular solderable pad was created with the LDS process to establish the electrical connection of the MID carrier and the flexible sensor chip.

To improve the mechanical stability between the sensor chip and the MID, four metallized pads with a diameter of

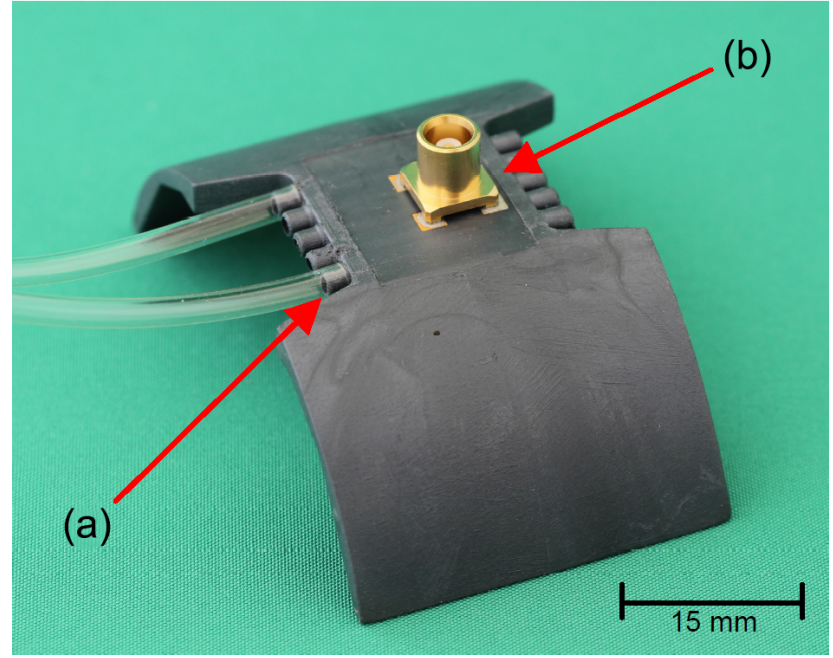

Figure 3. Back side of the 3D-MID carrier with connected fluidic tubes (a) and mounted MCX connector (b).

$2 \mathrm{~mm}$ were fabricated on the curved side areas of the LCP substrate, shown in Fig. 2.

In the middle of the main mounting sector, a central electric VIA was laser drilled from the top to the back side of the MID and filled during the electroless plating process to establish an electrical connection for the signal transfer to a standard MCX connector at the back side, shown in Figs. 2 and 3. A circular shielding pad around the central sensor via is connected on the front side of the MID with four additional metallized vias to the grounding pads of the MCX connector at the back side. These grounded contacts minimize the influence of external fields on the sensor signal between the sensor and the connector (Fig. 3).

\section{Fabrication of the sensor device}

The fabrication process of the sensor was divided into three parts (Fig. 4). The first fabrication processes were completed on a silicon wafer where the SU-8 microfluidic structures and the sensor bottom electrodes were defined, shown in Fig. 4 (Part 1). The first part starts with the cleaning of the $100 \mathrm{~mm}$ silicon wafer in a HF $1 \%$ dip for $1 \mathrm{~min}$. An adhesion layer of the Omnicoat ${ }^{\mathrm{TM}}$ was spun onto the clean substrate and baked for $1 \mathrm{~min}$ on a hotplate at $200^{\circ} \mathrm{C}$. In order to improve the releasing speed, the thickness of the Omnicoat ${ }^{\mathrm{TM}}$ layer was enlarged by subsequent multiple coating. Then, the handling SU-8-50 layer was coated on the Si wafer in a thickness of $50 \mu \mathrm{m}$ without any structures. After the first SU-8 layer coating, the bottom electrode made of titanium $(30 \mathrm{~nm})$ and aluminum $(300 \mathrm{~nm})$ was deposited on the polymer and structured via lift-off. The surface of the SU-8 was pretreated with a plasma activation step in a mixture of $\mathrm{O}_{2} / \mathrm{SF}_{6}$ to improve the adhesion between the polymer and metal electrodes. After stripping the photoresist layer, a $50 \mu \mathrm{m}$ thick 

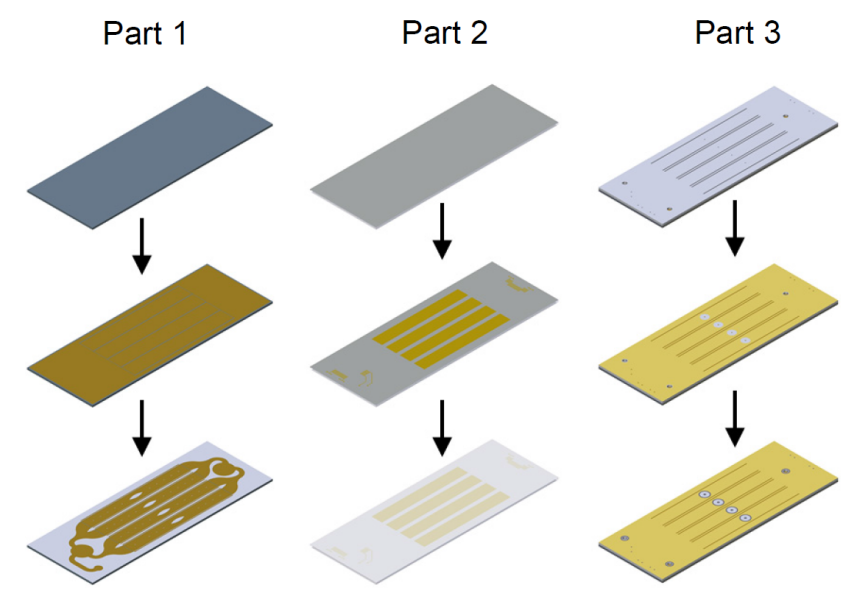

Figure 4. Fabrication of the complete sensor. Part 1: fabrication of the bottom electrode and the microfluidic channels of the sensor chip; part 2: manufacturing of the top electrode and the SU-8 top cap; part 3: establishment of the bonding, dry etching, and plating of the contact pad structures.

SU-8-50 layer was spin coated and structured to implement the microfluidic channels. To avoid any thermo-mechanical stress, all baking processes are needed to be temperature ramped. The parameters of the SU-8 processing also should be adjusted in order to obtain the flexible free-standing microfluidic structures. It is known that the softbake, exposure, and post exposure bake are the most influencing factors on the SU-8 layer mechanical properties. In order to complete the described microfluidic structure, the bake temperatures were lowered to $85^{\circ} \mathrm{C}$ and the exposure dose was reduced to $160 \mathrm{~mJ} \mathrm{~cm}^{-2}$.

The next part of the sensor manufacturing included the fabrication of the sensor top electrode structures on the SU8/glass wafer, shown in Fig. 4 (Part 2). A borosilicate glass was cleaned in a solution of $\mathrm{H}_{2} \mathrm{SO}_{4}$ and $\mathrm{H}_{2} \mathrm{O}_{2}$ at $135^{\circ} \mathrm{C}$ for $10 \mathrm{~min}$ and then coated with Omnicoat ${ }^{\mathrm{TM}}$ for the further releasing of the next $50 \mu \mathrm{m}$ thick layer of SU-8-50. An additional plasma treatment was performed to activate the surface of the polymer layer to improve the adhesion of the subsequently sputtered titanium and aluminum metal layers that were further lift-off structured. The SU-8/metal layer was further covered with a thin film of SU-8-5. After the covering of the metal structure, the silicon and the glass wafer were adhesive bonded under assistance of a SUSS MA6/BA6 mask aligner and a SUSS SB6e substrate bonder. Finally, after the bonding step the glass wafer was released in a wet etchant.

On top of the free SU-8 surface a $200 \mathrm{~nm}$ aluminum layer was sputtered and structured via lithography and etching. The Al mask was used in an Oxford Instruments PlasmaSystem 100 dry etching process to open the polymer layers and establish the entry for the top sensor electrode contact and the sidewalls for the shielding. To avoid any thermal mechanical stress in the SU-8 layers during the plasma etching process

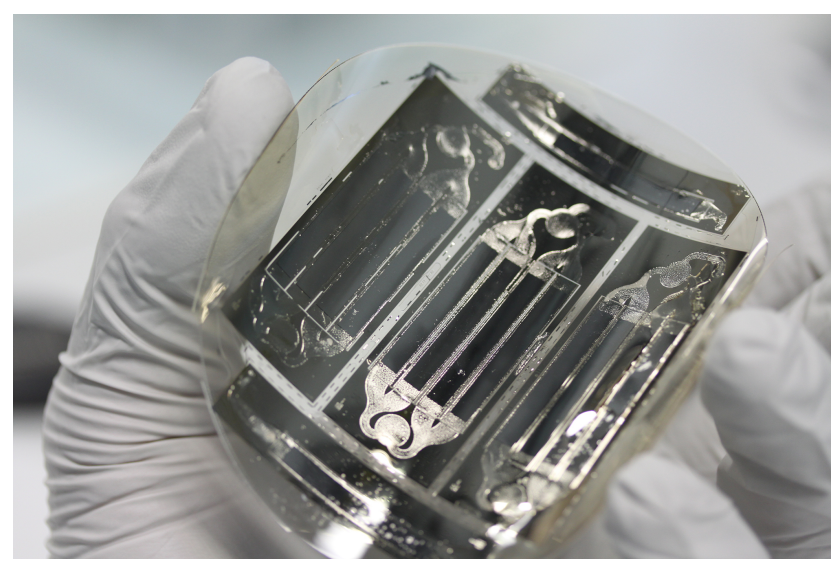

Figure 5. Completed SU-8-based free-standing impedance spectroscopy sensors with different designs on a $100 \mathrm{~mm}$ substrate.

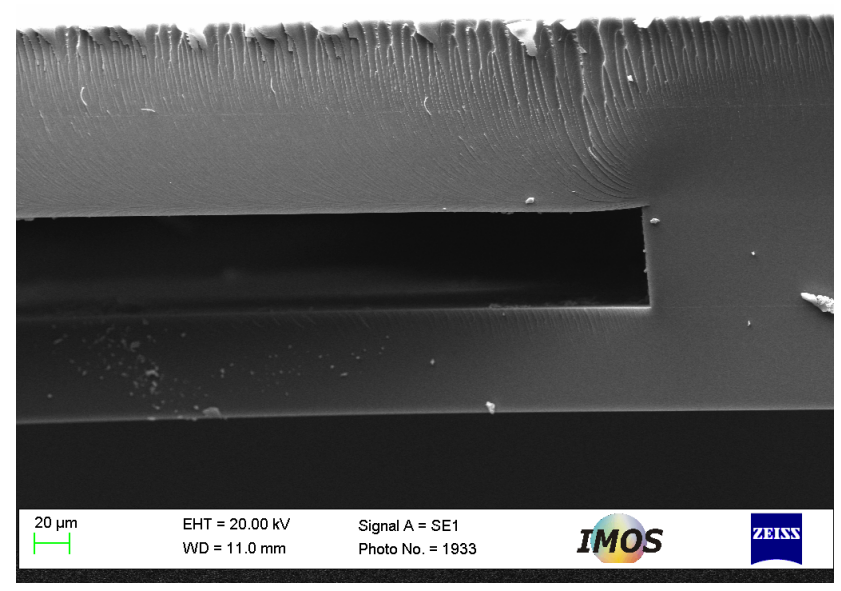

Figure 6. SEM cross-section image of a free-standing microfluidic channel.

with a mixture of $\mathrm{SF}_{6} / \mathrm{O}_{2}$, the $\mathrm{Si}$ handling wafer was backside cooled with helium and the etching process was divided into steps of $30 \mathrm{~s}$ etching and $120 \mathrm{~s}$ pause. After stripping the masking material, a new layer of $500 \mathrm{~nm} \mathrm{Al}$ was structured with a lift-off process to connect the top electrodes and establish the 3-D-shielding. To realize the solder connections, a final photoresist TI Spray was spray coated with a SUSS Delta Altaspray on the Al / SU-8 surface. The final layers of nickel and gold were plated on the contact pads by the use of an electroless metallization bath. After the metallization, the completed SU-8 multilayer structures were separated from the Si handling wafer in a releasing step of the Omnicoat ${ }^{\mathrm{TM}}$ layer with the help of the developer MF-319.

\section{Experimental results}

The fully processed free-standing microfluidic sensors intended for the further separation are shown in Fig. 5. The sensors are fabricated on a 4 in. wafer level and demonstrate 


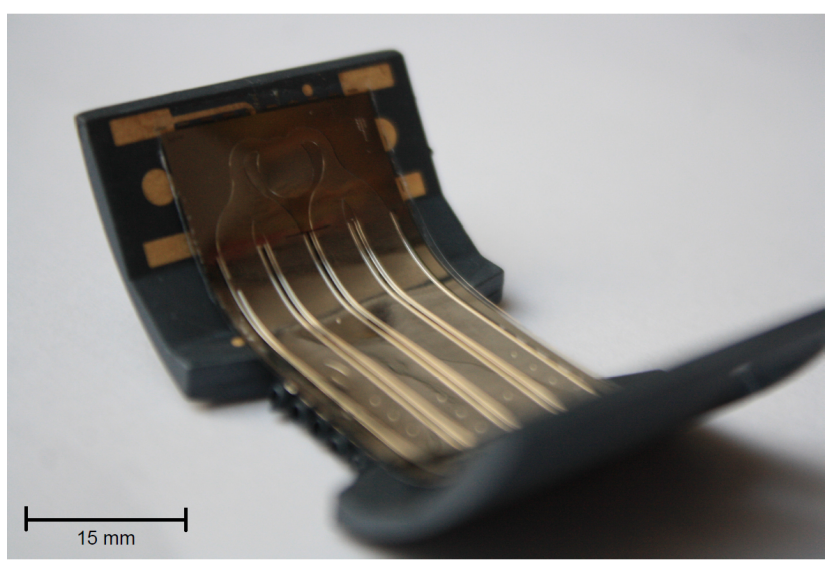

Figure 7. Microfluidic impedance spectroscopy sensor directly attached on the 3D-MID carrier.

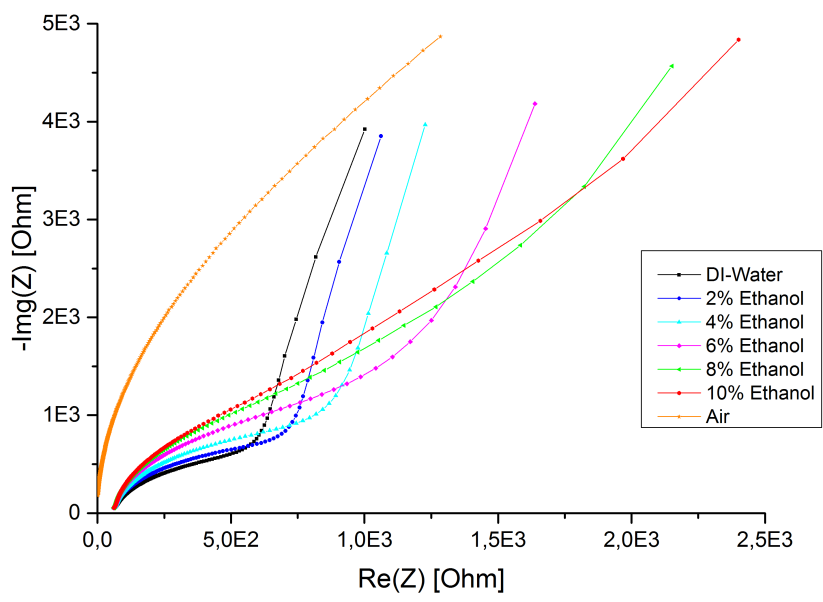

Figure 8. Impedance measurement of water, air, and a mixture of water and ethanol in a Cole-Cole plot for the frequency range of $10 \mathrm{kHz}-10 \mathrm{MHz}$.

a sufficient flexibility for an attachment on a 3-D substrate. A SEM cross-section view of the free-standing microfluidic channel is shown in Fig. 6. As it can be seen, there are no boundaries between the different SU-8 layers and the sidewalls are almost vertical. The bonding area is completely closed without any defects even after a sample dicing.

The self-developed 3D-MID platform was injection molded with the help of ARBURG 320s equipment. The 3DMID substrate, shown in Figs. 2 and 3, integrates all electrical as well as fluidic interconnections between the microfluidic sensor chip and the other devices that are necessary for the measurement.

Due to plated contact pads of the sensor, a direct attachment to the 3D-MID platform by a solder process without any additional chip wire bonding was possible. The microfluidic part of the 3D-MID was connected to a fluid system that contained a micropump, a reservoir, and a temperature con-
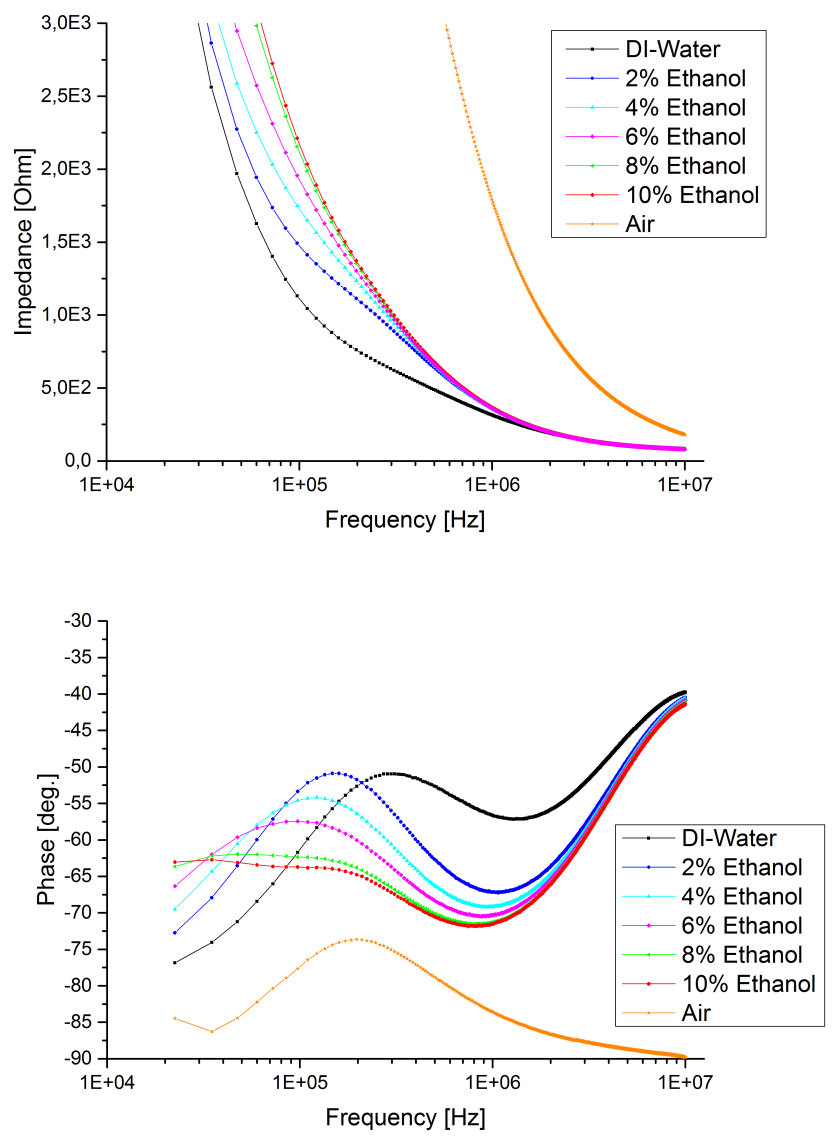

Figure 9. Impedance and phase measurement of water, air, and a mixture of water and ethanol in a series of Bode plots.

trol unit. All electrical ports of the MID were wired with an Agilent 4395A Network/Spectrum/Impedance analyzer.

The manufactured impedance spectroscopy sensor was experimentally investigated with the mixture of DI water and ethanol. The possible sensor application as an in-line sensor for ethanol-containing fuel analysis should be initially characterized with liquids that demonstrate a predictable behavior at the defined composition. Due to the complexity of gasoline compositions, its analysis with different ethanol content can provide a non-linear sensor response, whose explanation is beyond the scope of the current contribution.

The impedance measurements were conducted utilizing an Agilent 4395A Network/Spectrum/Impedance analyzer in "impedance analyzer" mode together with the impedance measuring set. The experimental results were obtained in an analyzer multichannel mode where an impedance magnitude and a phase as well as real and imaginary parts of the impedance were measured in parallel.

In Figs. 8 and 9 the results of the complex impedance and phase measurements are shown for the frequency range of $10 \mathrm{kHz}-10 \mathrm{MHz}$. All measurements were made at a constant temperature of $22^{\circ} \mathrm{C}$. After an initial test of the sensor behavior with air and deionized water to check the function of 
the system, pure ethanol was added to the DI water. In the concentration range of $2-10 \%$ of ethanol mixed in deionized water, a sufficient sensitivity could be demonstrated with the presented microfluidic sensor system. The expected significant impedance and phase shift can be observed in the ColeCole and Bode plots, shown in Figs. 8 and 9.

\section{Conclusions}

The demonstrated technology process for the manufacturing of free-standing SU-8 structures provides the possibility of fabricating SU-8-based completely free-standing microfluidic sensors. As it was shown, the standard metal liftoff process opens the possibility of depositing sensor electrodes on the SU-8 surface on the wafer level. The technology of "buried" metal electrodes in SU-8 provides reliable structures with considerably low-cost processing. A control of the SU-8 process parameter makes it possible to achieve flexible and mechanically stable structures that can be used for the attachment of flexible sensor chips on 3-D molded interconnection devices. A releasing step completed with the standard Omnicoat ${ }^{\mathrm{TM}}$ process established the opportunity for a considerably clean wet SU-8 releasing even with relatively large-scale structures. As it was demonstrated on Fig. 5, the whole 4 in. wafer was released without any structural damage. The microfluidic multichannel sensor was assembled on the 3D-MID platform, which integrated all electrical and fluidic interconnections. The measurement results of the fabricated sensor system demonstrated a high sensitivity to the ethanol concentration by analyzing a water-ethanol mixture.

Acknowledgements. The authors would like to thank the Federal Ministry of Education and Research (BMBF) for funding of the MicroSens project and all employees of the MEMS cleanroom team of the IMOS in Magdeburg for their help and valuable contribution.

Edited by: S. Schmid

Reviewed by: two anonymous referees

\section{References}

Agirregabiria, M., Blanco, F. J., Berganzo, J., Arroyo, M. T., Fullaondo, A., Mayoraa, K., and Ruano-Lópeza, J. M.: Fabrication of SU-8 multilayer microstructures based on successive CMOS compatible adhesive bonding and releasing steps, Lab on a chip, 5, 545-552, doi:10.1039/b500519a, 2005.

Barsoukov, E. and Macdonald, J. R.: Impedance Spectroscopy: Theory, Experiment and Applications, John Wiley \& Sons, Inc, Hoboken, NJ, USA, 2005.

Despont, M., Lorenz, H., Fahrni, N., Brugger, J., Renaud, P., and Vettiger, P.: High-aspect-ratio, ultrathick, negative-tone near-uv photoresist for MEMS applications, in: IEEE The Tenth Annual International Workshop on Micro Electro Mechanical Systems, An Investigation of Micro Structures, Sensors, Actuators, Machines and Robots, Nagoya, Japan, 26-30 January 1997, 518522, 1997.

Ezkerra, A., Fernández, L. J., Mayora, K., and Ruano-López, J. M.: Fabrication of SU-8 free-standing structures embedded in microchannels for microfluidic control, J. Micromech. Microeng., 17, 2264-2271, doi:10.1088/0960-1317/17/11/013, 2007.

Gawad, S., Schild, L., and Renaud, P. H.: Micromachined impedance spectroscopy flow cytometer for cell analysis and particle sizing, Lab on a chip, 1, 76-82, doi:10.1039/b103933b, 2001.

Gómez, R., Bashir, R., Sarikaya, A., Ladisch, M. R., Sturgis, J., Robinson, J. P., Geng, T., Bhunia, A. K., Apple, H. L., and Wereley, S.: Microfluidic Biochip for Impedance Spectroscopy of Biological Species, Biomed. Microdev., 3, 201-209, doi:10.1023/A:1011403112850, 2001.

Jo, B.-H., van Lerberghe, L. M., Motsegood, K. M., and Beebe, D. J.: Three-dimensional micro-channel fabrication in polydimethylsiloxane (PDMS) elastomer, J. Microelectromech. Syst., 9, 76-81, doi:10.1109/84.825780, 2000.

Leneke, T., Hirsch, S., and Schmidt, B.: A multilayer process for the connection of fine-pitch-devices on molded interconnect devices (MIDs), Circuit World, 35, 23-29, doi:10.1108/03056120910953286, 2009.

Lin, C.-H., Lee, G.-B., Chang, B.-W., and Chang, G.-L.: A new fabrication process for ultra-thick microfluidic microstructures utilizing SU-8 photoresist, J. Micromech. Microeng., 12, 590597, doi:10.1088/0960-1317/12/5/312, 2002.

Liu, C.: Recent Developments in Polymer MEMS, Adv. Mater., 19, 3783-3790, doi:10.1002/adma.200701709, 2007.

Lorenz, H., Despont, M., Fahrni, N., LaBianca, N., Renaud, P., and Vettiger, P.: SU-8: A low-cost negative resist for MEMS, J. Micromech. Microeng., 7, 121-124, doi:10.1088/09601317/7/3/010, 1997.

Moser, D. and Krause, J.: 3-D-MID - Multifunctional Packages for Sensors in Automotive Applications, in: Advanced Microsystems for Automotive Applications 2006, edited by: Valldorf, J. and Gessner, W., VDI-Buch, Springer-Verlag, Berlin/Heidelberg, 369-375, 2006.

Oseev, A., Zubtsov, M., and Lucklum, R.: Gasoline properties determination with phononic crystal cavity sensor, Sensors and Actuators B: Chemical, 189, 208-212, doi:10.1016/j.snb.2013.03.072, 2013.

Pesantez, D., Amponsah, E., and Gadre, A.: Wet release of multipolymeric structures with a nanoscale release 
layer, Sensors and Actuators B: Chemical, 132, 426-430, doi:10.1016/j.snb.2007.10.060, 2008.

Schmidt, M.-P., Leneke, T., Hirsch, S., and Schmidt, B.: A novel injection molded fluidic interposer for microfluidic applications, 4th Electronic System-Integration Technology Conference (ESTC), Amsterdam, Netherlands, 17-20 September 2012, 1-4, doi:10.1109/ESTC.2012.6542059, 2012.

Schmidt, M.-P., Oseev, A., Engel, C., Brose, A., Aman, A., and Hirsch, S.: A Novel Design and Fabrication of Multichannel Microfluidic Impedance Spectroscopy Sensor for Intensive Electromagnetic Environment Application, Procedia Engineering, 87, 88-91, doi:10.1016/j.proeng.2014.11.272, 2014a.

Schmidt, M.-P., Oseev, A., Engel, C., Brose, A., and Hirsch, S.: Impedance spectroscopy microfluidic multichannel sensor platform for liquid analysis, 18th International Conference on Miniaturized Systems for Chemistry and Life Sciences, San Antonio, Texas, USA, 26-30 October 2014, 2137-2139, doi:10.13140/2.1.4254.8004, 2014b.
Vilares, R., Hunter, C., Ugarte, I., Aranburu, I., Berganzo, J., Elizalde, J., and Fernandez, L. J.: Fabrication and testing of a SU-8 thermal flow sensor, Sensors and Actuators B: Chemical, 147, 411-417, doi:10.1016/j.snb.2010.03.054, 2010.

Wang, P., Tanaka, K., Sugiyama, S., Dai, X., and Zhao, X.: Wet releasing and stripping SU-8 structures with a nanoscale sacrificial layer, Microelectronic Engineering, 86, 2232-2235, doi:10.1016/j.mee.2009.03.079, 2009.

Zhang, J., Tan, K. L., Hong, G. D., Yang, L. J., and Gong, H. Q.: Polymerization optimization of SU-8 photoresist and its applications in microfluidic systems and MEMS, J. Micromech. Microeng., 11, 20-26, doi:10.1088/0960-1317/11/1/304, 2001. 